\title{
Laplace pressure based disjoining pressure isotherm in non symmetric conditions
}

Axel Huerre, Marie-Pierre Valignat, A. C. Maggs, Olivier Theodoly, and Marie-Caroline Jullien

Citation: Appl. Phys. Lett. 111, 221601 (2017); doi: 10.1063/1.4997857

View online: https://doi.org/10.1063/1.4997857

View Table of Contents: http://aip.scitation.org/toc/apl/111/22

Published by the American Institute of Physics

\section{Articles you may be interested in}

Dense arrays of site-controlled quantum dots with tailored emission wavelength: Growth mechanisms and optical properties

Applied Physics Letters 111, 221102 (2017); 10.1063/1.5004407

Terahertz birefringence and absorption of a chalcopyrite $\mathrm{CdSiP}_{2}$ crystal

Applied Physics Letters 111, 221103 (2017); 10.1063/1.5006660

High-efficiency and low-loss gallium nitride dielectric metasurfaces for nanophotonics at visible wavelengths Applied Physics Letters 111, 221101 (2017); 10.1063/1.5007007

High-gain monolithic 3D CMOS inverter using layered semiconductors

Applied Physics Letters 111, 222101 (2017); 10.1063/1.5004669

Ultra-transparent media with anisotropic mass density for broadband acoustic invisibility

Applied Physics Letters 111, 223501 (2017); 10.1063/1.5002741

Phase retrieval of an electron vortex beam using diffraction holography

Applied Physics Letters 111, 223101 (2017); 10.1063/1.4998595

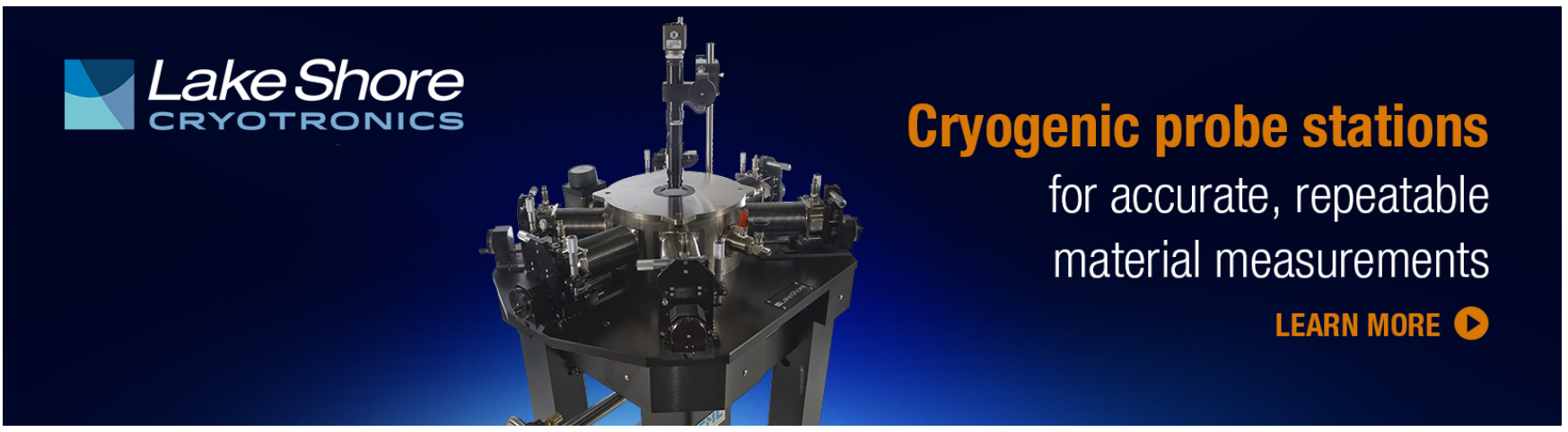




\title{
Laplace pressure based disjoining pressure isotherm in non symmetric conditions
}

\author{
Axel Huerre, ${ }^{1}$ Marie-Pierre Valignat, ${ }^{2}$ A. C. Maggs, ${ }^{1}$ Olivier Theodoly, ${ }^{2}$ \\ and Marie-Caroline Jullien ${ }^{1}$ \\ ${ }^{1}$ Gulliver UMR CNRS 7083, PSL research University, ESPCI Paris, 10 rue Vauquelin, F-75005 Paris, France \\ ${ }^{2}$ LAI, INSERM UMR S 1067, CNRS UMR 7333, Aix-Marseille Universite, 13009 Marseille, France
}

(Received 27 July 2017; accepted 9 November 2017; published online 27 November 2017)

\begin{abstract}
Understanding the stability and dynamics of two phase systems, such as foams and emulsions, in porous media is still a challenge for physicists and calls for a better understanding of the intermolecular interactions between interfaces. In a classical approach, these interactions are investigated in the framework of Derjaguin, Landau, Verwey, and Overbeek (DLVO) theory by building disjoining pressure isotherms. This paper reports on a technique allowing the measurement of disjoining pressure isotherms in a thin liquid film squeezed by either a gas or a liquid phase on a solid substrate. We couple a Reflection Interference Contrast Microscopy set-up to a microfluidic channel that sets the disjoining pressure through the Laplace pressure. This simple technique is found to be both accurate and precise. The Laplace pressure mechanism provides extremely stable conditions and offers opportunity for parallelizing experiments by producing several drops in channels of different heights. We illustrate its potential by comparing experimental isotherms for oil- $[$ (water and sodium dodecyl sulfate (SDS)] — glass systems with different models focusing on the electrostatic contribution of the disjoining pressure. The extracted values of the interface potentials are in agreement with the constant surface potential model and with a full computation. The derived SDS surface concentration agrees with values reported in the literature. We believe that this technique is suitable for investigating other working fluids and intermolecular interactions at smaller scales. Published by AIP Publishing. https://doi.org/10.1063/1.4997857
\end{abstract}

Fully understanding the forces at play in nanometric thin liquid films is critical to gain control of the stability and dynamics of foams, emulsions, and pseudoemulsions. This situation appears in applications as varied as enhanced oil recovery, ${ }^{1}$ cosmetics, ${ }^{2}$ or digital microfluidics, ${ }^{3}$ in which thin films of liquid are squeezed between a solid and a dispersed phase. In the following, we focus on a non-wetting dispersed phase such that a liquid film always develops between the solid substrate and the dispersed phase, be it liquid or gas. At rest, the stability and the resulting thickness of the film are set by the disjoining pressure stemming from a combination of several repulsive or attractive potentials. ${ }^{4-6}$ In the Derjaguin, Landau, Verwey, and Overbeek (DLVO) theory, the disjoining pressure is decomposed into structural $\left(P_{\mathrm{s}}\right)$, van der Waals $\left(P_{\mathrm{vdW}}\right)$, and electrostatic interactions $\left(P_{\mathrm{el}}\right)$. In the following, $P_{\mathrm{el}}$ will refer only to the entropic confinement contribution of the electrostatic potential..$^{5}$ The structural repulsion acts at distances smaller than $5 \mathrm{~nm}$, whereas vdW and electrostatic contributions develop over tens of nanometers. It has been shown recently that intermolecular forces operating at these nanoscopic scales strongly influence the dynamics of dispersed objects such as bubbles and drops confined at micrometric scales. ${ }^{7}$ Characterizing these forces is thus of strong importance to understand the dynamics of twophase systems. ${ }^{1,8,9}$ The direct measurement of the disjoining pressure isotherm for free-standing films (symmetric configuration) is widely reported with techniques such as the ThinFilm Balance (TFB), the micro bike-wheel, or the Film Trapping Technique. ${ }^{10-15}$ A surface force apparatus can be adapted to study liquid-liquid-solid configurations, but its use does not allow for gas-liquid-solid configurations. ${ }^{1,16}$ Consequently, fluid-liquid-solid configurations are commonly studied using a bubble or droplet mechanically pressed over a wetting film deposited on a substrate. ${ }^{17-20}$ The maximum disjoining pressure range experimentally achieved with this technique lies below $500 \mathrm{~Pa}$. A major limitation of those methods is the drainage time one has to wait between two measurement points as it can last several hours.

In this letter, we propose a technique allowing the characterization of disjoining pressure isotherms in a fluid-liquidsolid system simply requiring microfluidic chips and an inverted microscope equipped with the Reflection Interference Contrast Microscopy technique (RICM). Using microfluidics allows the investigation of higher disjoining pressures (here $3200 \mathrm{~Pa}$ ) and reduces fluctuations as the imposed pressure is controlled solely by the capillary pressure (set by the channel thickness). Additionally, droplets can be produced in several microfluidic chips and stored independently during the drainage time (which can reach a day for $10 \mathrm{~nm}$ thick film; $\propto h_{e q}^{-320}$ ) without monopolizing the microscope leading to the possibility of high throughout characterization. Moreover, the fluid used can be either a gas or a liquid. We believe that this technique is of high interest for the soft matter community working at microscales (microfluidics, porous media). We also question the possibility of extracting interfacial properties such as the potential of the two interfaces, solid-liquid and liquid-liquid, in an a priori non-symmetric case and show good agreement with a classical model.

In our system, the disjoining pressure is set by the confinement of a droplet in a micrometric Hele-Shaw cell. More 
precisely, a droplet is squeezed in a cavity of height $2 \mathrm{H}$ ranging from 10 to $481 \mu \mathrm{m}$, with an in plane radius $R \gg H$ such that the Laplace pressure leads to $\Delta P \sim \gamma / H$. At rest, the disjoining pressure developed in the thin film balances the Laplace pressure [see Fig. 1(b)]. Tuning the disjoining pressure thus simply leads to systems of various thicknesses, which in our experimental range leads to a disjoining pressure lying between 3200 and $80 \mathrm{~Pa}$. An important technical feature is that the pressure, set by the confinement, does not fluctuate. The film thickness is measured using RICM. ${ }^{21}$ The droplets are composed of mineral oil (SIGMA 8042-47-5), whereas the external phase is an aqueous solution of deionized water with Sodium Dodecyl Sulfate (SDS, SIGMA 15121-3) at $4.92 \mathrm{mM}$. The surface tension between the two fluids is $\gamma=1.6 \times 10^{-2} \mathrm{~N} \mathrm{~m}^{-1}$, measured with the pendant drop method. The two interfaces forming the film are thus glass/ water and water/oil. The substrate is a glass coverslip cleaned in a Piranha solution. Microchannels are molded using PDMS (Sylgard 184 Dow Corning). Glass coverslips and PDMS chips are finally bonded with an air plasma. Oilin-water droplets are generated at a T-junction in the microfluidic system and driven in the Hele-Shaw cell. Each design is scaled according to the cell's height as sketched in Fig. 1(a). Once the droplet reaches the center of the Hele-Shaw cell, the flow is stopped and the capillaries unplugged. The inlets and outlets are closed by pieces of glass slides to avoid any evaporation in the channels. We use the RICM technique fully described by Huerre et al. ${ }^{21}$ with the following parameters. The optical indexes are 1.515, 1.33, 1.467, and 1.412 for the glass plate, water-surfactant solution, mineral oil, and PDMS, respectively; illumination numerical apertures of 0.38 and 0.7 as well as illumination wavelengths of 450 , 546 , and $610 \mathrm{~nm}$ are used. The channel ceiling is found to contribute to the RICM signal. This influence is taken into account in the optical model by introducing a weighting of the channel ceiling contribution to the signal through a parameter $p$ defined in Ref. 21. The values given to $p$ obey the following rules: $p=1$ if $2 H<25 \mu \mathrm{m} ; p=100 / 75-1 /$ $75 * 2 H$ if $25<2 H<100 \mu \mathrm{m}$; and $p=0$ if $2 H>100 \mu \mathrm{m}$. The pixel resolution is $0.6 \mu \mathrm{m}$; the pictures are normalized by the background without a droplet to correct the inhomogeneities of illumination. The thickness $h$ of the wetting film is obtained by averaging the local thickness over a circular central zone where the film thickness is constant [see inset in

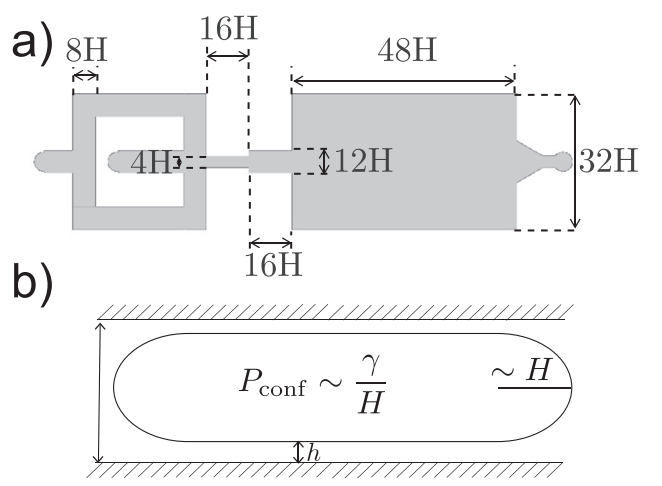

FIG. 1. (a) Schematics of the microfluidic chip and notation used in the text. (b) Laplace pressure set-up: droplet cross-section, the pressure inside the drop is in equilibrium with the disjoining pressure in the film of thickness $h$.
Fig. 2(a)]. The diaphragm is opened at a minimal value to avoid meniscus reflections. The intensity in the film is monitored until it reaches a constant value. For the thickest cells, the drainage time can be very long and the film is allowed to drain overnight.

Figure 2(a) displays the experimental isotherm where the disjoining pressure is given by the Laplace pressure, and the film thickness is measured using RICM. The error bar on the pressure stems from the uncertainty on the channel thickness $(\sim 0.2 \mu \mathrm{m})$. The error bar on the film thickness $h$ stems from the optical model, ${ }^{21}$ which in the present paper is at maximum $\pm 5 \mathrm{~nm}$ and at best $\pm 2 \mathrm{~nm}$ for a range of film thicknesses lying between 18 and $39 \mathrm{~nm}$. A positive disjoining pressure isotherm is the signature of a stable liquid film due to repulsive interactions between the two interfaces. In our experimental configuration, the Hamaker constant $A=6.7 \times 10^{-21} \mathrm{~J}$ is positive, i.e., the contribution of the $\mathrm{vdW}$ interaction between the two interfaces is attractive. With this value of Hamaker's constant, even for a solution containing $\mathrm{NaCl}$ at $0.4 \mathrm{M}$, a classical DLVO calculation shows that $\mathrm{vdW}$ interactions only play a significant role for films thinner than $0.6 \mathrm{~nm}$. We can thus consider the experimental curve being associated with the electrostatic contribution of the disjoining pressure. With our clean room facilities, droplets were found to be extremely sensitive to solid surface irregularities with triple line pinning and wetting for cell thicknesses below $10 \mu \mathrm{m}$. However, there is $a$ priori no limitation in investigating higher pressures, or correspondingly smaller thicknesses, apart from the microfabrication ones. One may thus explore smaller scale interactions. As pointed out in the introduction, knowing the shape

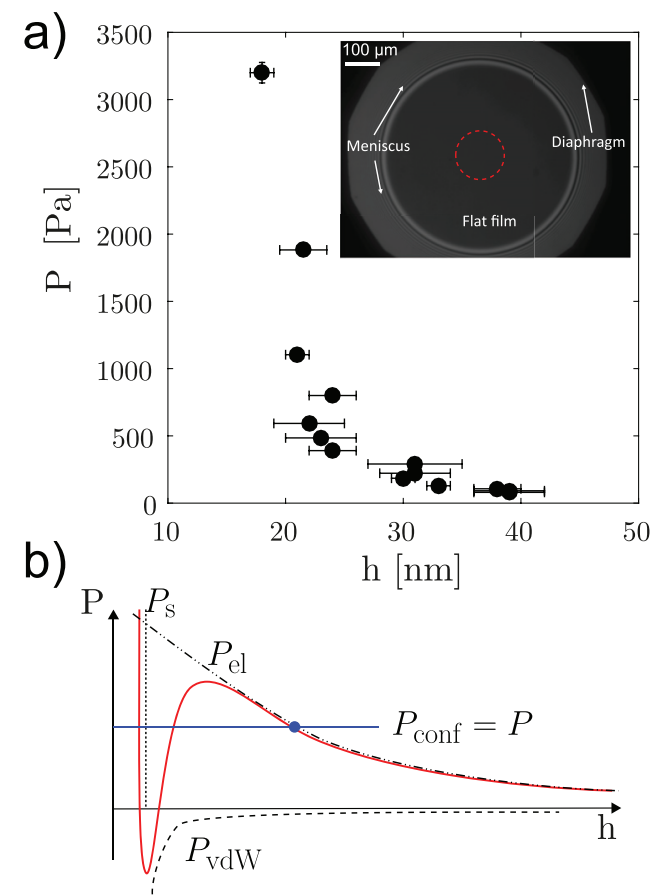

FIG. 2. (a) Typical disjoining pressure isotherm obtained with the Laplace pressure set-up. Different points are measured in cells of different thicknesses. Inset: typical raw image from which the flat film thickness is extracted (over the red dashed zone). (b) Disjoining pressure isotherm containing the steric, vdW, and electrostatic contributions. The height of the cell sets $P_{\text {conf }}$, allowing for the measurement of one point on the isotherm. 
of the isotherms makes it possible to deepen our understanding of the imbibition of diphasic fluids in microporous media. Finally, the proposed methodology is easy to set up for any fluid/liquid/solid configuration.

To go a step further, we discuss here the shape of the electrostatic contribution, relatively to the interface properties. A full modelling is out of the scope of this paper, and we rather stress the potential of the methodology. More precisely, as the configuration is not symmetric, it is possible to extract interface properties such as the apparent surface potentials $\Psi_{1}$ and $\Psi_{2}$ or equivalently the surface charges $\sigma_{1}$ and $\sigma_{2}$. Both surfaces interact through the water film with a Debye length $\kappa^{-1}=\sqrt{\epsilon k_{B} T / 2 e^{2} z^{2} C_{0}} \sim 4.4 \mathrm{~nm}$, with $\epsilon$ being the fluid permittivity, $z=1$ the valence of a SDS molecule, $e$ the charge of an electron, $C_{0}$ the SDS concentration, $k_{B}$ the Boltzmann constant, and $T$ the absolute temperature. In the following, we consider classical approaches ${ }^{5,22}$ to derive the disjoining pressure considering solely the entropic contribution of the electrostatic potential and neglecting the net Coulombic attraction. Several models are possible: (1) constant surface charge, (2) constant surface potential, and (3) Linear Superposition Approximation (LSA). In the following, the experimental data are fitted using these models, allowing us to extract surface charge values that are then compared with a full computation.

In the constant surface charge model, the disjoining pressure in the low potential limit (LPL) $\Psi_{i}<25 \mathrm{mV}^{5}$ up to $50 \mathrm{mV}^{22}$ is

$$
\begin{aligned}
P= & n k_{B} T\left[2\left(1+\frac{\left(y_{1}+y_{2}\right)^{2}}{4} \sinh ^{-2}(\kappa h / 2)\right)^{1 / 2}\right. \\
& \left.-\frac{\left(y_{1}-y_{2}\right)^{2} \exp (-\kappa h)}{1+\frac{1}{4}\left(y_{1}+y_{2}\right)^{2} \sinh ^{-2}(\kappa h / 2)}-2\right]
\end{aligned}
$$

where $y_{i}=e \Psi_{i} / k_{B} T$ are the reduced potentials for isolated plates, $h$ the film thickness, and $n$ the number of anions per unit volume.

In the constant surface potential case, considering that the potentials fulfill the LPL, the disjoining pressure is modeled by

$$
P=n k_{B} T\left[\left(2 y_{1} y_{2} \cosh (\kappa h)-y_{1}^{2}-y_{2}^{2}\right) / \sinh ^{2}(\kappa h)\right] .
$$

The LSA model, based on a weak overlap approximation, is less restrictive in the sense that for two symmetric plates, this is the potential at mid-plane $\Psi_{m}$ that is linearized in the LPL. No further assumption is made on the values of the potentials at the surface. Considering this, the disjoining pressure is

$$
P=64 n k_{B} T \gamma_{1} \gamma_{2} \exp (-\kappa h)
$$

with $\gamma_{i}=\tanh \left(y_{i} / 4\right)$. For two asymmetric surfaces, this model is still valid as long as there is a position in the gap between the plates where $\partial_{h} \Psi=0$.

These different models are fitted to the experimental data allowing for the reduced potentials to vary. The surface charges are then estimated using the Grahame equation ${ }^{5}$

$$
\sigma_{i}=\sqrt{8 \epsilon k_{B} T \rho_{\infty}} \sinh \left(e \Psi_{i} / 2 k T\right),
$$

where $\rho_{\infty}=n / \mathcal{N}_{A}$ is the SDS concentration in M, and $\mathcal{N}_{A}$ is the Avogadro constant.

Finally, we propose to compare the experimental data with a full computation of the pressure resulting from both the Coulombic and the entropic contributions of the electrostatic contribution. We first write the Poisson-Boltzmann equation considering the pressure ${ }^{5}$

$$
-P=\frac{\epsilon}{2}\left(\frac{d \Psi}{d h}\right)^{2}+2 k_{B} T \rho_{\infty}\left(1-\cosh \left(\frac{e \Psi}{k_{B} T}\right)\right) .
$$

We then consider a boundary condition with a constant charge $\sigma_{i}$ at each surface, $\epsilon\left(\frac{d \Psi_{i}}{d h}\right)=-\sigma_{i}$. Finally, after nondimensionalization, we obtain the integral formulation of the disjoining pressure isotherm $P(h)$

$$
\int \frac{d y}{\sqrt{-p-2(1-\cosh (\mathrm{y}))}}=\int d h^{\prime},
$$

where $p=P / k_{B} T \rho_{\infty}$ and $h^{\prime}=\kappa h$. This integral is solved numerically between the two interfaces with two different surface charges $\sigma_{1}$ and $\sigma_{2}$, varying $p$.

The experimental data are fitted by the different models in Fig. 3. Note that the models are symmetric in terms of indexes 1 and 2, which does not allow for the attribution of a given potential to a given surface. The solid lines correspond to the best fit for which the potentials $\Psi_{i}$ are the fitting parameters, summarized in Table I for the different models. The corresponding surface charges $\sigma_{i}$ are also indicated in the table. In order to evaluate the sensitivity of each model with the fitting parameters, surface charges $\sigma_{i}$ are allowed to vary in a range of $25 \%$ in the dark blue zone and in a range of $50 \%$ in the light blue zone (see supplementary material for the detailed method and minimization maps). Using this procedure, the experimental data lie within the error bars for all models. We can discard the constant surface charge model [Fig. 3(a)] as Eq. (1) is obtained considering the LPL which is not verified as $\left|\Psi_{2}\right|=146 \mathrm{mV}$. Furthermore, Kumar and Biswas ${ }^{16}$ report a glass surface potential in water lying between -45 and $-85 \mathrm{mV}$ for $\mathrm{pH}=6$. The approximations needed for the constant surface potential model [Fig. 3(b)] to remain valid can be considered fulfilled as both potentials are below $50 \mathrm{mV}$ in this scenario. However, fitting the experimental curve with (3) only allows us to extract $z=\gamma_{1} \gamma_{2}=0.225$ and not to extract single values for $\Psi_{1}$ and $\Psi_{2}$ (see supplementary material for a minimization map). Thus, the use of this model does not allow us to conclude on the values of the potential. Finally, the full computation [Fig. $3(d)$ ] leads to values of the same order of magnitude as the constant surface potential and also results in a symmetrical configuration. In light of this result, the LSA model for symmetric planes leads to surface potentials of $52 \mathrm{mV}$, which is in quantitative agreement with the FC. Considering the obtained surface charge of $\sigma_{1}=-1.3 \mu \mathrm{C} \mathrm{cm}^{-2}$ on the water/ oil interface and total charge dissociation, the surface concentration of SDS molecules is estimated to be $8 \times 10^{16}$ molecules $\mathrm{m}^{-2}$. With an ionization of $10 \%,{ }^{23}$ this value is 

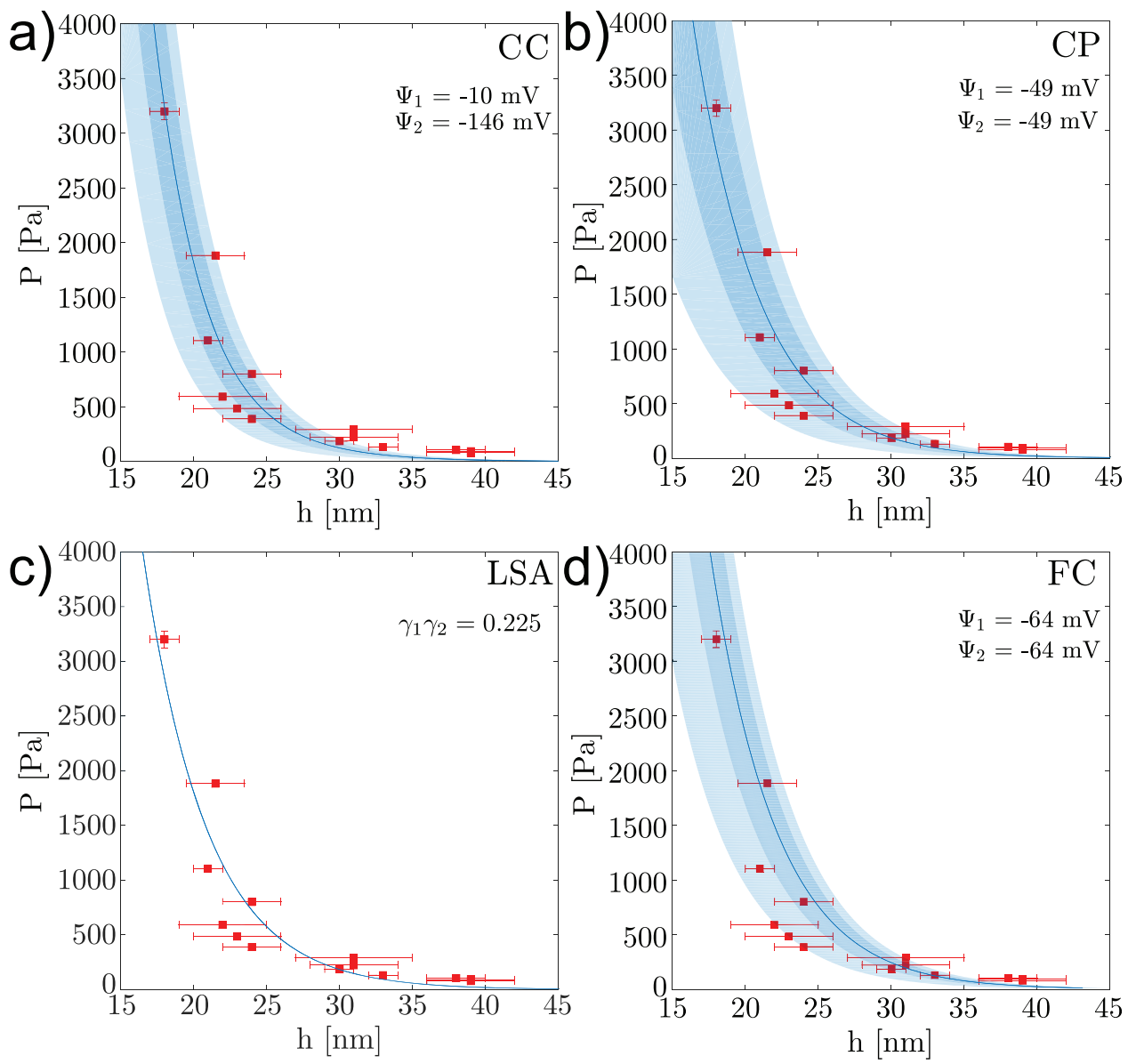

FIG. 3. Results from the fits for different models. The blue curve represents the optimized values of the potentials. The dark blue area represents a $25 \%$ variation of the surface charges and the light blue a 50\% variation. (a) Fit with the constant surface charge model (CC). (b) Fit with the constant surface potential model (CP). (c) Fit with the LSA model with $z=\gamma_{1} \gamma_{2}$ as a single fitting parameter. (d) Fit from the full calculation (FC).
TABLE I. Surface potentials given by the best fits, and surface charges, obtained by the different models. Error estimates of $25 \%$ and $50 \%$ are sketched in Fig. 3. Despite a best fit yielding symmetrical values for $\Psi_{1}$ and $\Psi_{2}$, satisfactory solutions are obtained for a large range of values of surface potential, for the second and third scenarios. See supplementary material.

\begin{tabular}{lcc}
\hline \hline Model & $\begin{array}{c}\text { Surface potential } \\
(\mathrm{mV})\end{array}$ & $\begin{array}{c}\text { Surface charge } \\
\left(\mu \mathrm{C} / \mathrm{cm}^{2}\right)\end{array}$ \\
\hline Constant surface charge, Eq. (1) & $\Psi_{1}=-10$ & $\sigma_{1}=-0.2$ \\
Constant surface potential, Eq. (2) & $\Psi_{2}=-146$ & $\sigma_{2}=-7.1$ \\
Full calculation Eq. (4) & $\Psi_{1}=-49$ & $\sigma_{1}=-0.9$ \\
& $\Psi_{2}=-49$ & $\sigma_{2}=-0.9$ \\
LSA Eq. (3) & $\Psi_{1}=-64$ & $\sigma_{1}=-1.3$ \\
\hline \hline
\end{tabular}

consistent with Pradines et al. ${ }^{24}$ finding of $1.2 \times 10^{18}$ molecules $\mathrm{m}^{-2}$. This leads to a charge condensation of $\sim 90 \%$ of the SDS molecules at the water/oil interface. On the other side, $\sigma_{2}=-1.3 \mu \mathrm{C} \mathrm{cm}^{-2}$ is consistent with a bare glass/water interface. $^{25}$

In conclusion, we propose an easy to implement methodology to measure disjoining pressure isotherms for fluidliquid-solid configurations. The pressure is set by the Laplace pressure, by simply squeezing a wetting film with a droplet or a bubble, allowing us to run experiments in parallel. The full set-up is reduced to a classical microfluidic set-up: a microfluidic chip to set the pressure and an inverted microscope equipped with RICM to measure the film thickness. In order to stress the reliability of our experimental approach, surface potentials and surface charges are extracted and found to be in good agreement with the literature using the constant surface potential model and a full computation method. This paper focused on a liquid dispersed phase, but the method is also suitable for the gaseous dispersed phase. This set up allows one to envision routine experiments with different working liquids both for applicative issues such as enhanced oil recovery and for more fundamental issues by reinforcing our understanding on intermolecular interactions.

See supplementary material for the procedures used to check the sensitivity of the models and the minimization map to extract the best fits.

This work was supported by CNRS, IPGG (Equipex ANR-10-EQPX-34), ESPCI Paris, Agence Nationale de la Recherche under the Grant No. 13-BS09-0011-01 and INSERM.

\footnotetext{
${ }^{1}$ S. Basu and M. M. Sharma, J. Colloid Interface Sci. 181, 443 (1996).

${ }^{2}$ D. Langevin, ChemPhysChem 9, 510 (2008).

${ }^{3}$ J. Kleinert, V. Srinivasan, A. Rival, C. Delattre, O. D. Velev, and V. K. Pamula, Biomicrofluidics 9, 034104 (2015).

${ }^{4}$ B. V. Derjaguin and E. V. Obukov, Acta Physicochim. URSS 5, 1 (1936).

${ }^{5} \mathrm{~J}$. N. Israelachvili, Intermolecular and Surface Forces (Academic press, 2011).

${ }^{6}$ N. V. Churaev, Russ. Chem. Rev. 73, 25 (2004).

${ }^{7}$ A. Huerre, O. Theodoly, A. M. Leshansky, M.-P. Valignat, I. Cantat, and M.-C. Jullien, Phys. Rev. Lett. 115, 064501 (2015).

${ }^{8}$ C. N. Baroud, F. Gallaire, and R. Dangla, Lab Chip 10, 2032 (2010).

${ }^{9}$ S. I. Karakashev, K. W. Stöckelhuber, R. Tsekov, and G. Heinrich, J. Colloid Interface Sci. 412, 89 (2013).

${ }^{10}$ A. Sheludko, Adv. Colloid Interface Sci. 1, 391 (1967).

${ }^{11}$ K. J. Mysels and M. N. Jones, Discuss. Faraday Soc. 42, 42 (1966).
} 
${ }^{12}$ A. Hadjiiski, R. Dimova, N. D. Denkov, I. B. Ivanov, and R. Borwankar, Langmuir 12, 6665 (1996).

${ }^{13}$ V. Bergeron, J. Phys.: Condens. Matter 11, R215 (1999).

${ }^{14}$ L. G. Cascão Pereira, C. Johansson, H. W. Blanch, and C. J. Radke, Colloids Surf. A 186, 103 (2001).

${ }^{15}$ C. Stubenrauch and R. von Klitzing, J. Phys.: Condens. Matter 15, R1197 (2003).

${ }^{16}$ D. Kumar and S. K. Biswas, J. Colloid Interface Sci. 348, 255 (2010).

${ }^{17}$ M. Aronson, M. Petko, and H. Princen, J. Colloid Interface Sci. 65, 296 (1978).

${ }^{18}$ R. G. Horn, M. Asadullah, and J. N. Connor, Langmuir 22, 2610 (2006).
${ }^{19}$ J. N. Connor and R. G. Horn, Faraday Discuss. 123, 193 (2003).

${ }^{20}$ L. Bluteau, M. Bourrel, N. Passade-Boupat, L. Talini, E. Verneuil, and F. Lequeux, Soft Matter 13, 1384 (2017).

${ }^{21}$ A. Huerre, M.-C. Jullien, O. Theodoly, and M.-P. Valignat, Lab Chip 16, 911 (2016).

${ }^{22}$ J. Gregory, J. Colloid Interface Sci. 51, 44 (1975).

${ }^{23}$ O. Theodoly, L. Cascao-Pereira, V. Bergeron, and C. Radke, Langmuir 21, 10127 (2005)

${ }^{24}$ V. Pradines, J. Krägel, V. B. Fainerman, and R. Miller, J. Phys. Chem. B 113, 745 (2009)

${ }^{25}$ T. W. Healy and L. R. White, Adv. Colloid Interface Sci. 9, 303 (1978). 